\title{
Clinical outcomes of first line FOLFIRINOX vs. gemcitabine plus nab-paclitaxel in metastatic pancreatic cancer at the Yale Smilow Hospital System
}

\author{
Timil Patel ${ }^{1}$, Joseph Miccio $^{2}$, Michael Cecchini ${ }^{1}$, Thejal Srikumar ${ }^{1}$, Stacey Stein ${ }^{1}$, Jeremy Kortmanksy ${ }^{1}$, \\ Kimberly Johung', Jill Lacy ${ }^{1}$ \\ ${ }^{1}$ Department of Internal Medicine (Medical Oncology), Yale School of Medicine, New Haven, CT, USA; ${ }^{2}$ Department of Therapeutic Radiology, \\ Yale School of Medicine, New Haven, CT, USA \\ Contributions: (I) Conception and design: T Patel, M Cecchini, J Lacy; (II) Administrative support: None; (III) Provision of study materials or \\ patients: T Patel, M Cecchini, J Lacy; (IV) Collection and assembly of data: T Patel, T Srikumar, M Cecchini, J Lacy; (V) Data analysis and \\ interpretation: All authors; (VI) Manuscript writing: All authors; (VII) Final approval of manuscript: All authors. \\ Correspondence to: Jill Lacy, MD. Department of Medical Oncology, Yale School of Medicine, 333 Cedar St., PO Box 208028, New Haven, CT 06520, \\ USA. Email: jill.lacy@yale.edu.
}

Background: FOLFIRINOX (FFX) and gemcitabine plus nab-paclitaxel (GN) are established first line therapies for metastatic pancreatic cancer (MPC). There are, however, no randomized controlled trials comparing FFX and GN in the first line setting and real-world data on their comparative effectiveness is limited. We aimed to evaluate the outcomes of patients with MPC who were treated with first line FFX and GN and to further characterize dose modifications, discontinuation rates due to treatment toxicity, and rates of hospitalizations while on treatment.

Methods: We manually abstracted data from the electronic medical records (EMR) system at Yale Smilow Hospital and Smilow Cancer Hospital Care Centers for patients with MPC treated with at least one cycle of first line FFX or GN from January 2011 to April 2019. Patients who received prior neoadjuvant or adjuvant FFX or GN and adjuvant gemcitabine less than 6 months prior to metastatic recurrence were excluded. The median time to treatment discontinuation (TTD) and overall survival (OS) were determined using KaplanMeier method.

Results: We identified 363 patients for analysis; 269 (74\%) patients were treated with FFX and 94 (26\%) with GN. Median TTD was 4.8 (IQR, 2.3-8.0) months in the FFX group compared to 3.4 (IQR, 1.3-5.7) months in the GN group ( $\mathrm{P}=0.0037)$. Median OS was 11.3 (95\% CI: 10.7-12.9) months in the FFX group and 7.0 (95\% CI: 6.0-8.7) months in the GN group $(\mathrm{P}<0.001)$. Initial dose modifications occurred in $264(98 \%)$ and $86(91 \%)$ of FFX and GN treated patients, respectively $(\mathrm{P}=0.001)$. While on treatment, $56(60 \%)$ of GNtreated patients had at least one hospitalization vs. 110 (41\%) in the FFX-group ( $\mathrm{P}=0.002)$. Treatment was discontinued due to chemotherapy toxicity in $26(10 \%)$ and $14(15 \%)$ among the FFX and GN cohorts, respectively $(\mathrm{P}=0.275)$.

Conclusions: Patients treated with first line FFX had increased survival and TTD compared to patients treated with GN despite increased dose modifications and similar rates of treatment discontinuation due to treatment-related toxicity. GN-treated patients were older and more likely to be hospitalized while on treatment. Further study evaluating comparative effectiveness between these two regimens is warranted.

Keywords: Metastatic pancreatic cancer (MPC); FOLFIRINOX (FFX); gemcitabine plus nab-paclitaxel (GN); survival

Submitted Apr 19, 2021. Accepted for publication Sep 29, 2021.

doi: 10.21037/jgo-21-202

View this article at: https://dx.doi.org/10.21037/jgo-21-202 


\section{Introduction}

Pancreatic cancer is the third leading cause of cancerrelated mortality in the United States and estimates suggest that by 2030 , it will become the second leading cause of cancer related deaths $(1,2)$. Although the treatment for localized disease is potentially curative with surgical resection followed by adjuvant therapy, only about $20 \%$ of patients have resectable disease at the time of diagnosis (3). Approximately $45 \%$ of patients present with metastatic disease and the prognosis remains poor with 5-year survival rates less than $5 \%$ (4). The mainstay treatment for patients with metastatic pancreatic cancer (MPC) remains systemic chemotherapy. The ACCORD trial demonstrated statistically superior overall survival (OS) with 5 -fluorouracil + folinic acid + irinotecan + oxaliplatin [FOLFIRINOX (FFX)] vs. gemcitabine alone (median, 11.1 vs. 6.8 months; $\mathrm{HR}=0.57 ; \mathrm{P}<0.001)(5)$. This was followed by the MPACT trial, which demonstrated significantly longer OS with gemcitabine plus nab-paclitaxel (GN) vs. gemcitabine alone in patients with MPC (median, 8.7 vs. 6.6 months; HR $=0.72 ; \mathrm{P}<0.001)(6)$. Both FFX and GN have since been approved by the Food and Drug Administration as potential first-line treatment options in patients with MPC with a good performance status (7).

No prospective comparative trial exists between FFX and GN, and cross-trial comparisons are flawed due to differences in baseline patient characteristics. For example, the ACCORD trial restricted enrollment to patients $<76$ years of age and ECOG performance status $<2$, while MPACT did not exclude patients based on age and allowed patients with an ECOG performance status of 2. There have been a number of nonrandomized, retrospective comparisons of FFX and GN in an attempt to elucidate differences in safety and effectiveness. Several retrospective analyses evaluating outcomes from a national cancer registry and institutional cohorts have found comparable survival and safety profiles between FFX and GN (8-17). These studies, however, were often small in size, lacked details regarding dose modifications, and offered no insight into rates of treatment related toxicities that led to hospitalization or discontinuation between the two regimens. A further understanding of treatment and cancerrelated hospitalizations while receiving FFX or GN would help oncologists determine the optimal first line regimen to administer to their patients. Thus, the objective of our retrospective review was to compare OS and time to treatment discontinuation (TTD) and to characterize dose modifications, discontinuation rates due to toxicity and rates of hospitalizations for patients with MPC receiving FFX or GN as initial therapy.

We present the following article in accordance with the STROBE reporting checklist (available at https://dx.doi. org/10.21037/jgo-21-202).

\section{Methods}

\section{Design}

This study was conducted in accordance with the Declaration of Helsinki (as revised in 2013). The study was approved by institutional ethics review board of Yale University. Because of the retrospective nature of the study, the requirement for informed consent was waived. We conducted a retrospective review of clinical outcomes in patients with MPC treated with first line FFX or GN at Yale Smilow Hospital and Smilow Cancer Hospital Care Centers from January 2011 to April 2019. Patients were excluded if they received alternative first line regimens, prior neoadjuvant FFX or GN and adjuvant gemcitabine less than 6 months prior to metastatic recurrence.

\section{Treatment}

Patients treated with at least one cycle of full or modified dose FFX or GN were included.

\section{Data}

Individual patient data was manually abstracted from the electronic medical records (EMR) including demographics, histology, prior therapy, initial and subsequent dose reductions, grade 3 or greater laboratory adverse events, number of hospitalizations while on first line treatment, TTD, OS, and second line treatment data. TTD was defined as time from first treatment with FFX or GN to discontinuation for any reason, and OS was defined as time from first treatment with FFX or GN to death or censored at last contact.

\section{Statistics analysis}

Baseline characteristic were compared between the FFX and GN cohorts. The $\chi^{2}$ or the Wilcoxon rank-sum test were used to evaluate for any differences in the cohorts for categorical and continuous variables, respectively. The 
Table 1 Demographics and patient characteristics

\begin{tabular}{|c|c|c|c|}
\hline Variables & FFX (n=269), n [\%] & GN (n=94), n [\%] & $P$ value \\
\hline Age & & & $<0.001$ \\
\hline$<76$ & 242 [90] & $67[71]$ & \\
\hline$\geq 76$ & 27 [10] & 27 [29] & \\
\hline Gender & & & $<0.017$ \\
\hline Female & $119[44]$ & $55[58]$ & \\
\hline Male & $150[56]$ & 39 [41] & \\
\hline Prior treatment & & & 0.169 \\
\hline None & 247 [92] & 85 [91] & \\
\hline Surgery + gemcitabine & $17[6]$ & $4[4]$ & \\
\hline Surgery alone & $5[2]$ & $5[5]$ & \\
\hline Histology & & & 0.192 \\
\hline Adenocarcinoma & 266 [99] & 92 [98] & \\
\hline Other & $3[1]$ & 2 [2] & \\
\hline Site of tumor & & & 0.197 \\
\hline Head & $100[37]$ & $28[30]$ & \\
\hline Non-head & 169 [63] & 66 [70] & \\
\hline \multicolumn{4}{|l|}{ Site of metastasis at $1 \mathrm{~L}$} \\
\hline Liver & 202 [75] & $71[76]$ & 0.932 \\
\hline Peritoneum & 55 [21] & $15[16]$ & 0.335 \\
\hline Lung & 35 [13] & $16[17]$ & 0.342 \\
\hline Lymph nodes & $41[15]$ & $9[10]$ & 0.170 \\
\hline
\end{tabular}

FFX, FOLFIRINOX; GN, gemcitabine plus nab-paclitaxel.

median TTD and OS were estimated using Kaplan-Meier method and compared between FFX and GN cohorts using the log-rank test. OS and TTD were also calculated in the subgroups of age $\geq 76$ and $<76$ and similarly compared via the log-rank test. Differences in the overall rates of toxicity and hospitalization were also compared between the FFX and GN cohorts using the $\chi^{2}$ test.

\section{Results}

\section{Patient characteristics}

Between January 2011 to April 2019, 363 patients with MPC were treated with first line FFX or GN at the Yale Smilow Cancer Hospital or Smilow Cancer Hospital Care Centers. There were 269 (74\%) patients treated with FFX and $94(26 \%)$ with GN. The demographics and disease characteristics at the initiation of first line treatment are shown in Table 1. At the start of first line therapy, the sites of metastatic disease were similar across both treatment arms.

Patients in the FFX group had a median age of 64 (IQR, $57-70)$ years compared with 69 (IQR, 62-77) years in the GN group $(\mathrm{P}<0.001)$. There was a significantly larger proportion of patients at or above the age of 76 receiving GN than FFX (29\% vs. $10 \% ; \mathrm{P}<0.001)$.

In terms of prior therapy, 332 (91\%) of patients received no prior therapy, $21(5 \%)$ received surgery plus adjuvant gemcitabine and $10(2 \%)$ received surgery alone.

\section{Treatment characteristics and efficacy}

FFX was delivered in 14-day cycles and the majority of 
Table 2 Treatment characteristics

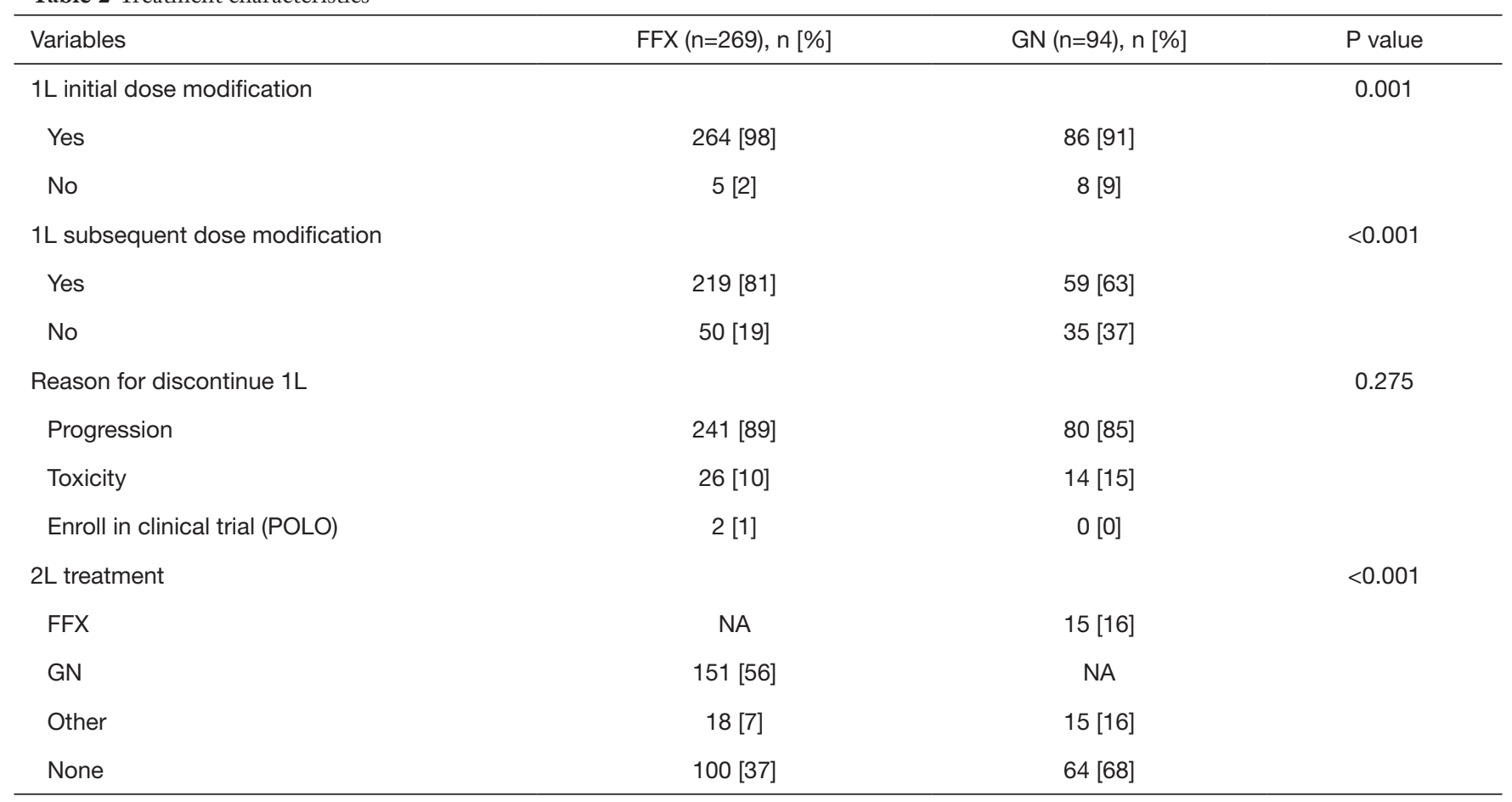

FFX, FOLFIRINOX; GN, gemcitabine plus nab-paclitaxel.

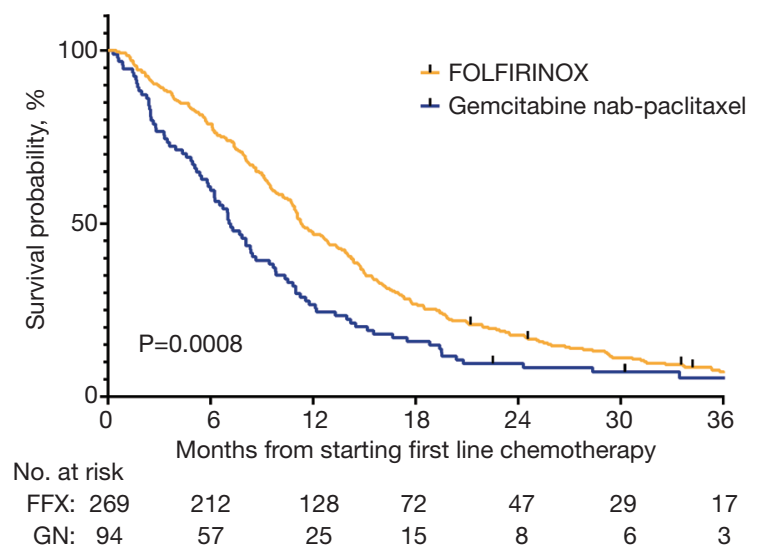

Figure 1 Kaplan-Meier curves of OS rates in MPC patients receiving FFX or GN. OS, overall survival; MPC, metastatic pancreatic cancer; FFX, FOLFIRINOX; GN, gemcitabine plus nab-paclitaxel.

patients at our institution receive the Yale modified regimen consisting of intravenous oxaliplatin $\left(85 \mathrm{mg} / \mathrm{m}^{2}\right)$, folinic acid $\left(400 \mathrm{mg} / \mathrm{m}^{2}\right)$, irinotecan $\left(135 \mathrm{mg} / \mathrm{m}^{2}\right), 5$-flurouracil $\left(300 \mathrm{mg} / \mathrm{m}^{2}\right)$ bolus and a 5 -flurouracil $\left(2,400 \mathrm{mg} / \mathrm{m}^{2}\right)$ continuous infusion for 46 hours followed by pegylated filgrastim on day 3 or 4 in the absence of severe leukocytosis (18).

At our institution GN was typically delivered in 28-day cycles with doses of nab-paclitaxel $\left(100 \mathrm{mg} / \mathrm{m}^{2}\right)$ followed by gemcitabine $\left(1,000 \mathrm{mg} / \mathrm{m}^{2}\right)$ on days 1,8 , and 15 . In the FFX cohort, $98 \%$ and $81 \%$ of pts had an initial and subsequent dose modification $(\mathrm{P}=0.001)$, respectively, compared to $91 \%$ and $63 \%$ in $\mathrm{GN}$ arm $(\mathrm{P}<0.001)$ (Table 2). The majority of the initial dose reduction was adhering to the Yale Modified FFX as outlined above.

The median follow-up period was 11.3 and 7.1 months in the FFX and GN groups, respectively, and the median follow up for patients alive as of April 2019 was 33.9 months. During the follow-up period, death occurred in $262(97 \%)$ and 89 (95\%) of patients who received FFX and $\mathrm{GN}$, respectively.

The median OS was 11.3 (95\% CI: 10.7-12.9) months in the FFX group and 7.0 (95\% CI: 6-8.7) months in the GN group $(\mathrm{P}<0.001)$ (Figure 1, Table 3). When survival was stratified by age, the median OS of patients under 76 was 11.7 months in the FFX and 7.1 months in the GN cohorts, respectively $(\mathrm{P}<0.001)$. On the other hand, the median OS of patients at or above the age of 76 was 8.0 months and 7.0 months in the FFX and GN cohorts, respectively ( $\mathrm{P}=0.8719)$ (Figure 2, Table 3). 
Table 3 Treatment efficacy

\begin{tabular}{|c|c|c|c|}
\hline Variables & $\operatorname{FFX}(\mathrm{n}=269)$ & GN $(n=94)$ & $P$ value \\
\hline \multicolumn{4}{|c|}{ Median TTD by age (months) } \\
\hline$<76$ & 5.2 & 4.2 & 0.004 \\
\hline$\geq 76$ & 3.2 & 3.7 & 0.440 \\
\hline \multicolumn{4}{|c|}{ Median OS by age (months) } \\
\hline$<76$ & 11.7 & 7.1 & 0.0007 \\
\hline$\geq 76$ & 8.0 & 7.0 & 0.8719 \\
\hline
\end{tabular}

FFX, FOLFIRINOX; GN, gemcitabine plus nab-paclitaxel; TTD, time to treatment discontinuation; OS, overall survival.

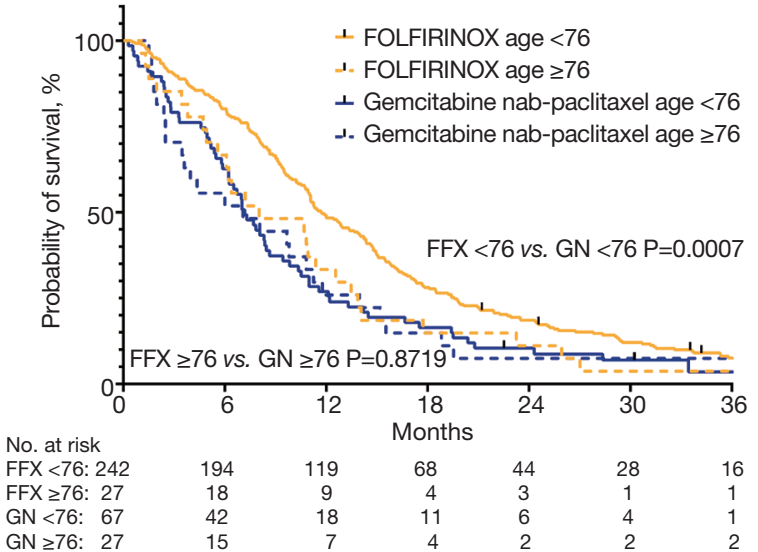

Figure 2 Kaplan-Meier curves of OS in MPC patients receiving FFX or GN stratified by age $\geq 76$ and $<76$. OS, overall survival; MPC, metastatic pancreatic cancer; FFX, FOLFIRINOX; GN, gemcitabine plus nab-paclitaxel.

The median TTD was 4.8 (IQR, 2.3-8.0) months in the FFX group compared to 3.4 (IQR, 1.3-5.7) months in the GN group, with FFX having a significantly longer chemotherapy duration than $\mathrm{GN}(\mathrm{P}=0.0037)$ (Figure 3, Table 3).

\section{Safety}

Treatment was discontinued due to chemotherapy toxicity in $26(10 \%)$ and $14(15 \%)$ among the FFX and GN cohorts, respectively $(\mathrm{P}=0.275)$. Table 4 characterizes the specific toxicity that led to discontinuation in each treatment group.

Patients initially treated with GN were more likely to be admitted to the hospital while on treatment $v s$. patients treated with FFX (60\% vs. $41 \% ; \mathrm{P}=0.002)$

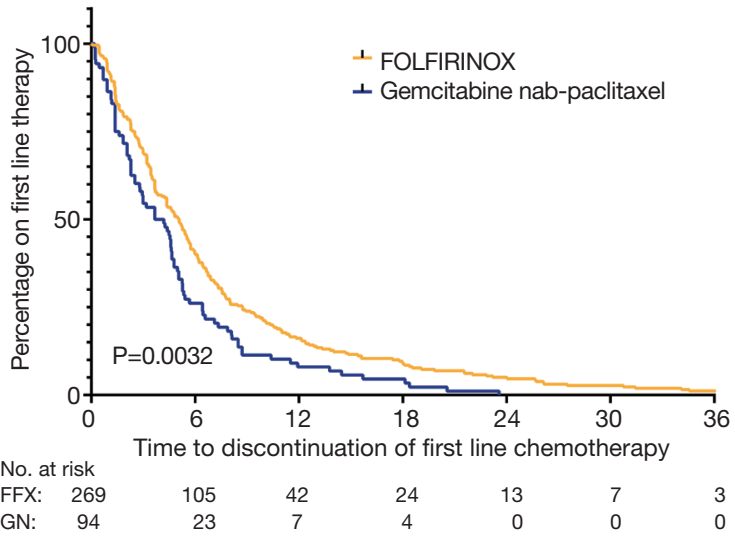

Figure 3 Kaplan-Meier curves of TTD rates in MPC patients receiving FFX or GN. TTD, time to treatment discontinuation; MPC, metastatic pancreatic cancer; FFX, FOLFIRINOX; GN, gemcitabine plus nab-paclitaxel.

(Table 5). Furthermore, patients on GN were also more likely to have multiple hospital admissions compared to the FFX cohort $(\mathrm{P}=0.033)$. The rates of treatment discontinuation due a hospitalization was similar between both FFX and GN cohorts (21\% vs. 23\%). Table 5 shows that the primary hospital admission diagnosis were similar across both treatment arms.

Table 5 characterizes the rates of hospital admission and the primary diagnosis that led to hospitalization among the two treatment groups as determined by admission and discharge notes.

There was no significant difference in the incidence of grade 3 or greater neutropenia, anemia or thrombocytopenia between the two groups (Table 5). The most common 
Table 4 Primary toxicity that led to treatment discontinuation

\begin{tabular}{lcc}
\hline Toxicity & FFX $(\mathrm{n}=26)$ & $\mathrm{GN}(\mathrm{n}=14)$ \\
\hline Sepsis & 9 & 0 \\
Diarrhea & 7 & 2 \\
Nausea, vomiting & 3 & 3 \\
Fatigue & 1 & 2 \\
Altered mental status & 2 & 1 \\
Pain & 0 & 1 \\
Other* & 4 & 5 \\
\hline
\end{tabular}

*, other (FFX vs. GN): hyperglycemia (1 vs. 0); gastric outlet obstruction (1 vs. 0); heart failure (0 vs. 1); hypoxic respiratory failure (0 vs. 2); hyperbilirubinemia ( 1 vs. 0$)$; pneumonitis (1 vs. 0 ); small bowel obstruction (0 vs. 2). FFX, FOLFIRINOX; GN, gemcitabine plus nab-paclitaxel.

treatment-related grade 3 or greater laboratory adverse event was thrombocytopenia, which occurred in $37(14 \%)$ and $12(13 \%)$ in the FFX and GN groups, respectively.

\section{Discussion}

This study represents one of the largest single center studies evaluating outcomes for MPC patients treated with FFX or GN with access to the source data allowing a high degree of clinical annotation. Our study is also the first published "real world" retrospective study to clearly demonstrate statistically superior median OS of first line FFX compared to GN (11.3 vs. 7.0 months; $\mathrm{P}<0.001)$. Furthermore, we report that patients treated with first line FFX had longer treatment duration compared to GN (TTD, 4.8 vs. 3.4 months; $\mathrm{P}=0.0037)$. These results are despite FFX treated patients having more initial and subsequent dose modifications compared to GN treated patients.

Although a comparative analysis of the PRODIGE and MPACT trials suggest that FFX may be associated with better OS compared to GN, the majority of retrospective reviews have not demonstrated superiority of FFX. Pacheco-Barcia et al. described increased survival with FFX compared to GN (14.0 vs. 7.0 months; $\mathrm{P}=0.02$ ) at the 2019 ESMO annual meeting but have not published their findings to date (19). In contrast, a metanalysis that included sixteen retrospective studies comparing safety and effectiveness of GN $v$ s. FFX and found no statistically significant difference in OS, progression-free survival (PFS), and toxicities between FFX and GN (20).
However, a metanalysis of retrospective studies is limited by unbalanced patient populations, differing treatment durations, and lack of access to the source data. A separate study utilizing data from the Navigating Cancer database reported similar survival and TTD in 202 patients treated with either FFN or GN (median TTD, 3.4 vs. 3.8 months; $\mathrm{P}=0.947$ ) (10). However, the study assessed survival by database persistence which is a proxy for but not a true measure of survival (median database persistence, $8.6 \mathrm{vs}$. 8.6 months; $\mathrm{P}=0.534$ ). Database persistence is an imperfect proxy because actions such as switching oncologists or leaving the EMR system due to hospice enrollment could have underestimated survival. Cartwright et al. published one of the largest retrospectives, multi-site, observational cohorts of patients with MPC treated with first line FFX $(\mathrm{n}=159), \mathrm{GN}(\mathrm{n}=255)$ and found no difference in time to treatment failure (TTF) or OS between the two groups (9). The authors, however, defined TTF as chemotherapy discontinuation for any reason but further insights into why treatment was discontinued are not provided. Furthermore, the study initially started with 2,901 patients, but the authors excluded 2,415 (83\%) patients due to reasons such as lacking documentation of site of metastasis, missing histology information and clinical trial enrollment. Given that manual data abstraction and review was not done, the authors may have excluded a significant portion of patients that could have been included in the analysis, thus possibly introducing bias and distorting the survival results. Our retrospective study stands apart because we included all continuous MPC patients who met the inclusion criteria while also manually reviewing and abstracting individual patient data, thus allowing us to find the reasons for treatment discontinuation. We provide a high degree of quality control that allows for accurate data and thus reliable interpretation of the results.

Our study demonstrated that younger patients were more likely to be treated with FFX than GN. The survival was superior with FFX in patients younger than 76 compared to GN (11.7 vs. 7.1 months; $\mathrm{P}<0.001)$, with no statistical difference in survival amongst the two treatment arms when patients were over the age of 76 (8.0 vs. 7.0 months; $\mathrm{P}=0.871$ ). In a pan-European questionnaire sent to practicing oncologists, it was found that oncologists considered FFX to more toxic than GN (21). The selection bias for FFX in younger, more fit patients has been described in several larger retrospective studies as well, suggesting oncologists are forgoing FFX in older patients due to concerns for increased toxicity $(10,13,14)$. 


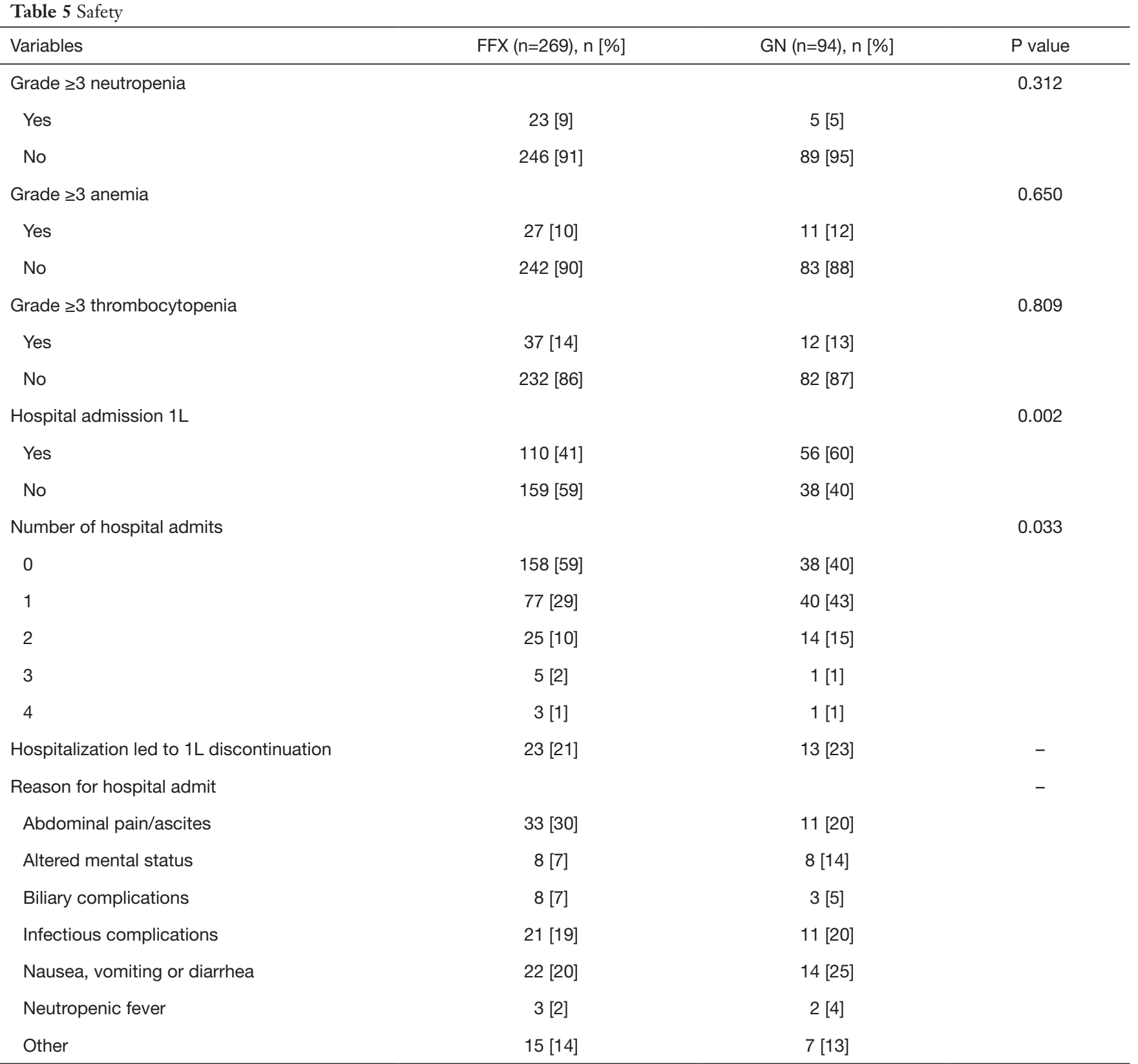

FFX, FOLFIRINOX; GN, gemcitabine plus nab-paclitaxel.

Our study, however, demonstrated no major differences in grade $\geq 3$ or higher hematologic adverse events between the two regimens. Furthermore, we also found that rates of treatment discontinuation for progression or toxicity to chemotherapy were similar between both arms, despite the GN cohort being older at the time of initiating therapy. We interpret these results as suggestive that dose modified FFX is a safe and effective regimen to consider in geriatric patients.
We are also the first to report that patients treated with first line GN had higher incidence of initial and recurrent hospitalizations while on treatment compared to FFX $(\mathrm{P}=0.002)$. This was also shown in another retrospective study by Chun et al., where they found interruptions of chemotherapy due to toxicity was more frequent in the GN group compared to FFX (29.3\% vs. 6.8\%; $\mathrm{P}<0.001)$ (22). We identified $41 \%$ of patients treated with FFX that had at least one hospitalization while on treatment compared 
to $60 \%$ of patients treated with $\mathrm{GN}(\mathrm{P}=0.002)$. Reported primary diagnoses for admission such as dehydration, constipation, respiratory failure, altered mental status and heart failure were more common reasons for a hospital admission in the GN group compared to the FFX group. Interestingly, we did not see a major difference in rates of treatment discontinuation due to hospitalizations, a surrogate for treatment related toxicity, among the two treatment cohorts. These findings may be reflective of an older patient population more commonly receiving first line GN who also presumably have increased comorbidities and fragility. Our findings regarding comparable toxicity and reduced hospitalizations between the two treatment arms despite FFX having more initial and subsequent dose modifications were unexpected because of the assumption that FFX is more toxic. Reduced hospitalization rates among the FFX treated patients, however, may be a consequence of increasing familiarity with the FFX regimen over the past decade leading to enhanced supportive care measures and anticipatory dose modifications to prevent toxicity. Our findings of comparable survival between FFX and GN in older adults $(\geq 76)$, however, needs to be further studied as perhaps the toxicities of treating older patients with FFX may be overstated and actually lead to decreased hospitalization while on treatment, thus improving quality of life.

Patients initially treated with FFX had a longer TTD than those treated with GN perhaps led to greater tumor burden reduction, which is known to be critical in improving survival in MPC. Furthermore, the higher rates of receiving second line treatment and lower incidence of hospitalizations in the FFX cohort could also explain the OS difference in favor of FFX, implying that FFX treated patients were younger and more fit. On the other hand, it is possible that patients treated with GN were sicker given their advanced age compared to the average FFX treated patient, thus the chances of receiving second line treatment was lower in this cohort. It is important to point out, however, that we do not know whether starting treatment with FFX rather than GN prevented rapid progression and/ or clinical decline thus leading to increased opportunities for second line therapies.

We also describe more common FFX use than GN in our overall study populations, which is contrast to national prescribing patterns. In community practice GN was more frequently prescribed as described by Cartwright $e t$ al. in a multi-site, retrospective cohort of 486 patients treated in community cancer centers around the United States where GN was more commonly prescribed in $52 \%$ of first line patients compared to $33 \%$ with FFX and 15\% with gemcitabine monotherapy (9).

At present, patients with MPC only have two first line treatment options. Thus, it is imperative to determine which MPC patients should receive FFX vs. GN. The strength of our study lies in the large population size of patients with MPC and manually abstracted patient level data. Data collected from the EMR represent actual treatment information documented as part of routine clinical care, and therefore demonstrate real-world clinical treatment patterns and outcomes as opposed to data obtained or collected as part of a controlled clinical trial or cancer registry databases. The limitations of this study include its retrospective design and being conducted at a single institution and the potential bias if there were blank fields or errors in the EMR database. Missing data cannot confirm the absence of a condition or value in patients' medical histories, only that it was not documented.

\section{Conclusions}

Patients treated with first line FFX had increased survival and TTD compared to patients treated with GN. FFXtreated patients were younger, less likely to be admitted to the hospital while on treatment and more likely to receive second line therapy compared to patients treated with GN. The rates of treatment discontinuation due to toxicity were similar between the FFX and GN cohorts, calling into question common assumptions regarding the tolerability of FFX. The median OS of patients treated with FFX and GN were similar to historical controls in randomized controlled trials, suggesting FFX should be considered for first line therapy in appropriately selected patients. Further study evaluating comparative effectiveness between these two regimens is warranted.

\section{Acknowledgments}

Funding: None.

\section{Footnote}

Reporting Checklist: The authors have completed the STROBE reporting checklist. Available at https://dx.doi. org/10.21037/jgo-21-202

Data Sharing Statement: Available at https://dx.doi. 
org/10.21037/jgo-21-202

Peer Review File: Available at https://dx.doi.org/10.21037/ jgo-21-202

Conflicts of Interest: All authors have completed the ICMJE uniform disclosure form (available at https:// dx.doi.org/10.21037/jgo-21-202). MC owns equity and serves on the scientific advisory board for Parthenon Therapeutics and receives consulting fees from Eisai Inc, Agio Therapeutic, and AstraZeneca. SS receives consulting fees from QED Therapeutics, Bristol-Myers Squibb, Bayer, Eisai, Merck and Genetech. JL receives consulting fees from Fresenius Kabi, Deciphera, Ipsen Biopharmaceuticals, Merck and Celgene/Bristol-Myers Squibb. The other authors have no conflicts of interest to declare.

Ethical Statement: The authors are accountable for all aspects of the work in ensuring that questions related to the accuracy or integrity of any part of the work are appropriately investigated and resolved. This study was conducted in accordance with the Declaration of Helsinki (as revised in 2013). The study was approved by institutional ethics review board of Yale University. Because of the retrospective nature of the study, the requirement for informed consent was waived.

Open Access Statement: This is an Open Access article distributed in accordance with the Creative Commons Attribution-NonCommercial-NoDerivs 4.0 International License (CC BY-NC-ND 4.0), which permits the noncommercial replication and distribution of the article with the strict proviso that no changes or edits are made and the original work is properly cited (including links to both the formal publication through the relevant DOI and the license). See: https://creativecommons.org/licenses/by-nc-nd/4.0/.

\section{References}

1. Siegel RL, Miller KD, Jemal A. Cancer statistics, 2019. CA Cancer J Clin 2019;69:7-34.

2. Rahib L, Smith BD, Aizenberg R, et al. Projecting cancer incidence and deaths to 2030: the unexpected burden of thyroid, liver, and pancreas cancers in the United States. Cancer Res 2014;74:2913-21.

3. Ducreux M, Cuhna AS, Caramella C, et al. Cancer of the pancreas: ESMO Clinical Practice Guidelines for diagnosis, treatment and follow-up. Ann Oncol 2015;26
Suppl 5:v56-68.

4. Sohal DPS, Kennedy EB, Khorana A, et al. Metastatic pancreatic cancer: ASCO Clinical Practice Guideline update. J Clin Oncol 2018;36:2545-56.

5. Conroy T, Desseigne F, Ychou M, et al. FOLFIRINOX versus gemcitabine for metastatic pancreatic cancer. $\mathrm{N}$ Engl J Med 2011;364:1817-25.

6. Von Hoff DD, Ervin T, Arena FP, et al. Increased survival in pancreatic cancer with nab-paclitaxel plus gemcitabine. N Engl J Med 2013;369:1691-703.

7. Tempero MA. NCCN guidelines updates: pancreatic cancer. J Natl Compr Canc Netw 2019;17:603-5.

8. Wang Y, Camateros P, Cheung WY. A real-world comparison of FOLFIRINOX, gemcitabine plus nabpaclitaxel, and gemcitabine in advanced pancreatic cancers. J Gastrointest Cancer 2019;50:62-8.

9. Cartwright TH, Parisi M, Espirito JL, et al. Clinical outcomes with first-line chemotherapy in a large retrospective study of patients with metastatic pancreatic cancer treated in a US community oncology setting. Drugs Real World Outcomes 2018;5:149-59.

10. Braiteh F, Patel MB, Parisi M, et al. Comparative effectiveness and resource utilization of nab-paclitaxel plus gemcitabine vs FOLFIRINOX or gemcitabine for the first-line treatment of metastatic pancreatic adenocarcinoma in a US community setting. Cancer Manag Res 2017;9:141-8.

11. Cho IR, Kang H, Jo JH, et al. FOLFIRINOX vs gemcitabine/nab-paclitaxel for treatment of metastatic pancreatic cancer: Single-center cohort study. World J Gastrointest Oncol 2020;12:182-94.

12. Franco F, Camara JC, Martín-Valadés JI, et al. Clinical outcomes of FOLFIRINOX and gemcitabine-nab paclitaxel for metastatic pancreatic cancer in the real world setting. Clin Transl Oncol 2021;23:812-9.

13. Kang J, Hwang I, Yoo C, et al. Nab-paclitaxel plus gemcitabine versus FOLFIRINOX as the first-line chemotherapy for patients with metastatic pancreatic cancer: retrospective analysis. Invest New Drugs 2018;36:732-41.

14. Kim S, Signorovitch JE, Yang H, et al. Comparative effectiveness of nab-paclitaxel plus gemcitabine vs FOLFIRINOX in metastatic pancreatic cancer: a retrospective nationwide chart review in the United States. Adv Ther 2018;35:1564-77.

15. Lee JC, Woo SM, Shin DW, et al. Comparison of FOLFIRINOX and gemcitabine plus nab-paclitaxel for treatment of metastatic pancreatic cancer: using Korean pancreatic cancer $(\mathrm{K}-\mathrm{PaC})$ registry. Am J Clin Oncol 
2020;43:654-9.

16. Papneja N, Zaidi A, Chalchal H, et al. Comparisons of outcomes of real-world patients with advanced pancreatic cancer treated with FOLFIRINOX versus gemcitabine and nab-paclitaxel: a population-based cohort study. Pancreas 2019;48:920-6.

17. Chiorean EG, Cheung WY, Giordano G, et al. Realworld comparative effectiveness of nab-paclitaxel plus gemcitabine versus FOLFIRINOX in advanced pancreatic cancer: a systematic review. Ther Adv Med Oncol 2019;11:1758835919850367.

18. Stein SM, James ES, Deng Y, et al. Final analysis of a phase II study of modified FOLFIRINOX in locally advanced and metastatic pancreatic cancer. Br J Cancer 2016;114:737-43.

19. Pacheco-Barcia V, France T, Zogopoulos G, et al. Gemcitabine plus nab-paclitaxel versus modified

Cite this article as: Patel T, Miccio J, Cecchini M, Srikumar T, Stein S, Kortmanksy J, Johung K, Lacy J. Clinical outcomes of first line FOLFIRINOX $v$ s. gemcitabine plus nab-paclitaxel in metastatic pancreatic cancer at the Yale Smilow Hospital System. J Gastrointest Oncol 2021;12(6):2547-2556. doi: 10.21037/ jgo-21-202
FOLFIRINOX as first line chemotherapy in metastatic pancreatic cancer: a comparison of toxicity and survival. Ann Oncol 2018;29:V46.

20. Pusceddu S, Ghidini M, Torchio M, et al. Comparative effectiveness of gemcitabine plus nab-paclitaxel and FOLFIRINOX in the first-line setting of metastatic pancreatic cancer: a systematic review and meta-analysis. Cancers (Basel) 2019;11:484.

21. Le N, Vinci A, Schober M, et al. Real-world clinical practice of intensified chemotherapies for metastatic pancreatic cancer: results from a pan-European questionnaire study. Digestion 2016;94:222-9.

22. Chun JW, Lee SH, Kim JS, et al. Comparison between FOLFIRINOX and gemcitabine plus nab-paclitaxel including sequential treatment for metastatic pancreatic cancer: a propensity score matching approach. BMC Cancer 2021;21:537. 